PROCEEDINGS OF THE

AMERICAN MATHEMATICAL SOCIETY

Volume 49, Number 1, May 1975

\title{
ZEROS OF POLYNOMIALS OVER FINITE
}

\section{PRINCIPAL IDEAL RINGS}

\section{MURRAY MARSHALL AND GARRY RAMAGE}

ABSTRACT. Let $R$ be a finite commutative ring with identity. For $f \epsilon$ $R\left[X_{1}, \cdots, X_{n}\right]$ denote by $N(f)$ the number of zeros of $f$ in $R^{(n)}$. For integers $n, d \geq 1$ denote by $A_{n, d}$ the greatest common divisor of the integers $N(f) ; f \in R\left[X_{1}, \cdots, X_{n}\right], \operatorname{deg} f=d$. J. Ax has shown that if $R$ is a field, then $A_{n, d}=|R|^{a}$ where $a$ is the integer satisfying $a<n / d \leq a+1$. In this paper, $A_{n, d}$ is computed in the case that $R$ is a principal ideal ring.

Throughout, let $R$ denote a finite commutative ring with identity, and let $R\left[X_{1}, \cdots, X_{n}\right]$ denote the ring of polynomials in $n$ variables over $R$, $n \geq 1$. Let $f \in R\left[X_{1}, \cdots, X_{n}\right]$. An element $x=\left(x_{1}, \cdots, x_{n}\right) \in R^{(n)}$ is called a zero of $f$ if $f(x)=0$. Let $N(f)=N(f, R)$ denote the total number of zeros of $f$ in $R^{(n)}$. Let $A_{n, d}=A_{n, d}(R)$ denote the greatest common divisor of the integers $N(f) ; f \in R\left[X_{1}, \ldots, X_{n}\right]$, deg $f=d$.

Suppose $\operatorname{deg} f=1$. Then $f=f_{0}+f_{1}$ where $f_{0}=f(0)$, and where $f_{1}$ is linear when considered as a function from $R^{(n)}$ to $R$. Thus, either $N(f)=0$ (if $f_{0} \notin \operatorname{Im} f_{1}$ ) or $N(f)=\left|\operatorname{Kern} f_{1}\right|$. But $\left|\operatorname{Kern} f_{1}\right|=\left|R^{(n)}\right| /\left|\operatorname{Im} f_{1}\right|$. It follows easily from this that $A_{n, 1}=|R|^{n-1}$. More generally, $A x$ [2] has shown that if $R$ is a field, then $A_{n, d}=|R|^{\alpha}$ where $\alpha$ is the integer defined by $\alpha$ $<n / d \leq a+1$. In this paper, we compute $A_{n, d}$ in case $R$ is a principal ideal ring.

1. First assume $R$ is a local principal ideal ring. Let $q$ denote the order of the residue field of $R$, and define $k$ by $q^{k}=|R|$. Assume $R$ is not a field, i.e. that $k \geq 2$. Let $\pi$ be a prime element of $R$. The following version of Hensel's lemma is useful:

Lemma. Suppose $g(x)=r_{0}+r_{1} X+\cdots+r_{d} X^{d} \in R[X]$ is such that $\pi \mid r_{i}, i=2, \cdots, d, \pi \nmid r_{1}$. Then $g$ has a unique zero in $R$.

Proof. $x_{1}=-r_{0} / r_{1}$ is the unique zero of $g$ modulo $\pi$. By induction, if $x_{i}$ is the unique zero of $g$ modulo $\pi^{i}$, then $x_{i+1}=x_{i}-g\left(x_{i}\right) / r_{1}$ is the unique

Received by the editors July 30, 1973.

AMS (MOS) subject classifications (1970). Primary 12C05, 13F 10. 
zero of $g$ modulo $\pi^{i+1}$. Since $\pi^{k}=0$, this implies $x_{k}$ is the unique zero of $g$ in $R$.

By this lemma, $A_{n, d}=1$ for $n=1$. Hence, we may assume $n, d \geq 2$. The computation of $A_{n, d}$ in this case is covered by the following

Theorem. Suppose $R$ is a finite local principal ideal ring which is not a field; notations as above. If $n, d \geq 2$, then $A_{n, d}=q^{\beta}$ where $\beta$ is the integer defined by $\beta<n k / 2 \leq \beta+1$.

Proof. Let $f \in R\left[X_{1}, \cdots, X_{n}\right], \operatorname{deg} f=d$. Partition the zeros of $f$ in $R^{(n)}$ into classes by considering two zeros $\left(x_{1}, \ldots, x_{n}\right),\left(x_{1}^{\prime}, \ldots, x_{n}^{\prime}\right)$ to be equivalent if $x_{i} \equiv x_{i}^{\prime} \bmod \pi$ for $i=1, \ldots, n$. We show $q^{\beta} \mid N(f)$ by showing $q^{\beta}$ divides the number of zeros in each class. Let $\left(x_{1}, \cdots, x_{n}\right)$ be a zero of $f$. Note that $f\left(x_{1}+\pi X_{1}, \cdots, x_{n}+\pi X_{n}\right)$ is of the form $\pi g_{1}+\pi^{2} g_{2}+$ $\cdots+\pi^{d} g_{d}$ where $g_{i} \in R\left[X_{1}, \cdots, X_{n}\right]$ is homogeneous of degree $i, i=1$, $\cdots, d$. Thus we wish to count the number of zeros of

$$
g=g_{1}+\pi g_{2}+\cdots+\pi^{d-1} g_{d}
$$

in $\left(R / \pi^{k-1}\right)^{(n)}$.

If $k=2, q^{\beta} \mid N\left(g, R / \pi^{k-1}\right)$ by Ax's result since $g=g_{1}$. If one of the coefficients of $g_{1}$ is a unit, we may assume, without loss of generality, that it is the coefficient of $x_{1}$. Let $y_{2}, \cdots, y_{n} \in R / \pi^{k-1}$ be arbitrary and let $h(X)=g\left(X, y_{2}, \cdots, y_{n}\right)$. Then by the Lemma, $h$ has a unique zero in $R / \pi^{k-1}$. Hence $N\left(g, R / \pi^{k-1}\right)=q^{(n-1)(k-1)}$. Thus

$$
\begin{aligned}
q^{\beta} \mid N\left(g, R / \pi^{k-1}\right) & \Leftrightarrow(n-1)(k-1) \geq \beta \\
& \Leftrightarrow(n-1)(k-1) \geq n k / 2-1 \\
& \Leftrightarrow(n-2)(k-2) \geq 0
\end{aligned}
$$

which is true since $n, k \geq 2$ by assumption.

If, on the other hand, $\pi$ divides all the coefficients of $g_{1}$, write $g_{1}=$ $\pi g_{1}^{\prime}$, and let

$$
g^{\prime}=g_{1}^{\prime}+g_{2}+\pi g_{3}+\cdots+\pi^{d-2} g_{d}
$$

Then $N\left(g, R / \pi^{k-1}\right)=N\left(g^{\prime}, R / \pi^{k-2}\right) q^{n}$. By induction (or by Ax's result, if $k=3), N\left(g^{\prime}, R / \pi^{k-2}\right)$ is divisible by $q^{\gamma}, \gamma<n(k-2) / 2 \leq \gamma+1$. Hence $N\left(g, R / \pi^{k-1}\right)$ is divisible by $q^{n+\gamma}$. But $n+\gamma=\beta$. Thus $q^{\beta} \mid N(f)$ for every $f \in R\left[X_{1}, \ldots, X_{n}\right]$ of degree $d$, so $q^{\beta} \mid A_{n, d}$.

We deal with the reverse divisibility in several steps. First note that $A_{n, d}$ is a power of the characteristic of the residue field of $R$. For example, 
consider any polynomial $f \in R\left[X_{1}, \cdots, X_{n}\right]$ of the form $f=f_{0}+f_{1}+\pi f_{2}+$ $\cdots+\pi f_{d}$ where $f_{i}$ is homogeneous of degree $i$ and where at least one of the coefficients of $f_{1}$ is a unit. Applying the Lemma as before, $f$ has $q^{(n-1) k}$ zeros, so $A_{n, d} \mid q^{k(n-1)}$.

We now complete the case $d=2$ by constructing, for each integer $n \geq 2$, a polynomial $f \in R\left[X_{1}, \cdots, X_{n}\right]$ of degree 2 such that $N(f) \equiv-q^{\beta} \bmod q^{\bar{\beta}+1}$. If $n=2$ take $f=X_{1} X_{2}-1$. Then $N(f)=q^{k}-q^{k-1} \equiv-q^{k-1} \bmod q^{k}$. For $n=2 t, t \geq 2$ define

$$
f=X_{1} X_{t+1}+X_{2} X_{t+2}+\cdots+X_{t} X_{2 t}
$$

For $0<s \leq k$ there are $q^{s t}-q^{(s-1) t}$ tuples $\left(x_{1}, \ldots, x_{t}\right)$ such that $R x_{1}$ $+\cdots+R x_{t}=R \pi^{k-s}$, and for each such tuple, the linear function $\left(y_{1}, \cdots\right.$, $\left.y_{t}\right) \rightarrow x_{1} y_{1}+\cdots+x_{t} y_{t}$ maps $R^{(t)}$ onto $R \pi^{k-s}$, so has $q^{k t-s}$ solutions. Thus

$$
N(f)=q^{k t}+\sum_{s=1}^{k}\left(q^{s t}-q^{(s-1) t}\right) q^{k t-s} .
$$

For $t \geq 2$, this is congruent to $-q^{k t-1}$ modulo $q^{k t}$.

For $n=2 t+1, t \geq 1$ take

$$
f=X_{1} X_{t+1}+\cdots+X_{t} X_{2 t}+\pi^{\epsilon} X_{2 t+1}^{2}
$$

where $\epsilon=0$ or 1 according to whether $k$ is even or odd. For $0<s \leq k$ there are

$$
\left(q^{s t}-q^{(s-1) t}\right) q^{(k+\epsilon) / 2+\delta(s)}
$$

tuples $\left(x_{1}, \cdots, x_{t}, x\right)$ satisfying

$$
R x_{1}+\cdots+R x_{t}=R \pi^{k-s} \supseteq R \pi^{\epsilon} x^{2},
$$

where $\delta(s)$ is the greatest integer $\leq s / 2$. For each such tuple, $x_{1} y_{1}+\cdots+$ $x_{t} y_{t}+\pi^{\epsilon} x^{2}=0$ has $q^{k t-s}$ solutions $\left(y_{1}, \ldots, y_{t}\right)$. Thus

$$
N(f)=q^{(k+\epsilon) / 2} q^{k t}+\sum_{s=1}^{k}\left(q^{s t}-q^{(s-1) t}\right) q^{(k+\epsilon) / 2+\delta(s)} q^{k t-s} .
$$

For $t \geq 1$ this is congruent to $-q^{(k+\epsilon) / 2+k t-1}$ modulo $q^{(k+\epsilon) / 2+k t}$.

To handle the case $d \geq 3$ write $d=2 r+s$ where $s=0$ or 1 and define

$$
g\left(X_{1}, \cdots, X_{n}\right)=f\left(X_{1}, \cdots, X_{n}\right)+\pi^{k-1} f\left(X_{1}, \cdots, X_{n}\right)^{r} X_{1}^{s},
$$

where $f$ is the polynomial of degree two constructed in the previous step. Then $g$ has the same zeros as $f$, and $g$ has degree $d$, so the proof is complete. 
Remark. Let $R$ be a local principal ideal ring. It follows from the above formulae for $A_{n, d}$ (or from Ax's formula if $k=1$ ) that $A_{n, d} \mid A_{n, d^{\prime}}$ if $d \geq d^{\prime}$. We will see later that this holds even when $R$ is not local.

2. Now let $R$ be any finite commutative principal ideal ring with identity. Then $R$ decomposes canonically as $R=R_{1} \oplus \cdots \oplus R_{s}$ where the $R_{i}$ are local principal ideal rings $[1$, p. 90$]$. This decomposition induces a natural isomorphism

$$
R\left[X_{1}, \cdots, X_{n}\right] \cong \bigoplus_{i=1}^{s} R_{i}\left[X_{1}, \cdots, X_{n}\right]
$$

and if $f \in R\left[X_{1}, \ldots, X_{n}\right]$ decomposes as $\left(f_{1}, \ldots, f_{s}\right)$ under this isomorphism, then $\operatorname{deg} f=\max \left\{\operatorname{deg} f_{i}: i=1, \cdots, s\right\}$ and $N(f, R)=\Pi_{i=1}^{s} N\left(f_{i}, R_{i}\right)$. It follows from this, together with the remark, that

$$
\Lambda_{n, d}(R)=\prod_{i=1}^{s} A_{n, d}\left(R_{i}\right) .
$$

This completes the computation of $A_{n, d}$ for $R$ any finite commutative principal ideal ring with identity.

\section{REFERENCES}

1. M. F. Atiyah and I. G. MacDonald, Introduction to commutative algebra, Addison-Wesley, Reading, Mass., 1969. MR 39 \#4129.

2. J. Ax, Zeroes of polynomials over finite fields, Amer. J. Math. 86 (1964), 255-261. MR $28 \# 3986$.

DEPARTMENT OF MATHEMATICS, UNIVERSITY OF SASKATCHEWAN, SASKATOON, CANADA S7N OWO 